\title{
S-Ketamine-Induced NMDA Receptor Blockade during Natural Speech Production and Its Implications for Formal Thought Disorder in Schizophrenia: A Pharmaco-fMRI Study
}

\author{
Arne Nagels, ${ }^{*, 2}$, Maurice Cabanis ${ }^{3,4}$, Andrea Oppel', Andre Kirner-Veselinovic ${ }^{5}$, Christian Schales' and \\ Tilo Kircher' \\ 'Department of Psychiatry and Psychotherapy, Philipps-University Marburg, Marburg, Germany; ${ }^{2}$ Department of English and Linguistics, Johannes \\ Gutenberg University, Mainz, Germany; ${ }^{3}$ Department of Psychiatry and Psychotherapy, Social Neuroscience Lab, University of Lübeck, Lübeck, \\ Germany; ${ }^{4}$ Clinic for Addiction Medicine and Addictive Behaviour, Centre for Mental Health, Stuttgart, Germany; ${ }^{5}$ Department of Psychiatry and \\ Psychotherapy, RWTH Aachen University, Aachen, Germany
}

Structural and functional changes in the lateral temporal language areas have been related to formal thought disorder (FTD) in schizophrenia. Continuous, natural speech production activates the right lateral temporal lobe in schizophrenia, as opposed to the left in healthy subjects. Positive and negative FTD can be elicited in healthy subjects by glutamatergic NMDA blockade with ketamine. It is unclear whether the glutamate system is related to the reversed hemispheric lateralization during speaking in patients. In a double-blind, crossover, placebo-controlled study, 15 healthy, male, right-handed volunteers overtly described 7 pictures for 3 min each while BOLD signal changes were acquired with fMRI. As a measure of linguistic demand, the number of words within $20 \mathrm{~s}$ epochs was correlated with BOLD responses. Participants developed S-ketamine-induced psychotic symptoms, particularly positive FTD. Ketamine vs placebo was associated with enhanced neural responses in the right middle and inferior temporal gyri. Similar to a previous fMRI study in schizophrenia patients vs healthy controls applying the same design, S-ketamine reversed functional lateralization during speech production in healthy subjects. Results demonstrate an association between glutamatergic imbalance, dysactivations in lateral temporal brain areas, and FTD symptom formation.

Neuropsychopharmacology (2018) 43, I324-1333; doi:I0.1038/npp.20 I7.270; published online 24 January 2018

\section{INTRODUCTION}

According to DSM-5 and ICD-10, formal thought disorder (FTD) is a core symptom of schizophrenia. On the level of neurotransmission the glutamate system has been regarded as one key to explain the pathophysiology of schizophrenia (Javitt, 2015; Kircher et al, in press). In healthy participants, administration of glutamatergic $N$-methyl-D-aspartate receptor (NMDAR) antagonists, such as phencyclidine and ketamine, induces acute psychosis, particularly FTD, negative symptoms, and cognitive impairments similar to those typically observed in schizophrenia (Abi-Saab et al, 1998; Adler et al, 1998; Kantrowitz and Javitt, 2010). In ketamine challenge experiments with healthy subjects, positive FTD (derailment, loosening of associations, neologisms, etc) as well as negative FTD (poverty of speech, circumstantiality, etc) have been elicited (Adler et al, 1999). Ketamine, in

* Correspondence: Professor Dr A Nagels, Department of Psychiatry and Psychotherapy, Philipps-University Marburg, Rudolf-BultmannStrasse 8, 35039 Marburg, Germany, Tel: +49 642I 58 65837, Fax: +496421 58 68939, E-mail: arne.nagels@staff.uni-marburg.de Received 25 April 2017; revised 14 September 2017; accepted 16 October 2017; accepted article preview online 6 November 2017 particular S-ketamine, thus induces FTD symptoms in healthy individuals that are highly comparable to those observed in schizophrenia patients.

On a structural brain level, FTD in schizophrenia has been related to a decreased lateralization of lateral temporal (superior temporal gyrus (STG) and middle temporal gyrus (MTG)) gray matter volume. Healthy subjects have a larger cortical temporal volume in the $\mathrm{LH}$; in patients this asymmetry is absent or decreased (Oertel-Knochel et al, 2012). These structural alterations correspond to lateral temporal lobe dysactivations in fMRI language experiments in patients. For example, in a naturalistic speech production approach, patients were asked to speak in the MR scanner freely about Rorschach inkblots (Kircher et al, 2001a, 2002a). A positive correlation between the amount of speech produced and STG/MTG activation in the LH for control subjects and in the RH for schizophrenia patients was found (Kircher et al, 2002a). Accordingly, FTD patients recruited the right 'Wernicke area' homolog that confirmed a longstanding assumption (Griesinger, 1861) that a reduced functional laterality or a perturbed hemispheric interaction is associated with FTD in schizophrenia (Horn et al, 2010; Kircher et al, 2002a; Oertel-Knochel et al, 2012). This loss of 
functional brain asymmetry resp. a lateral temporal lobe dysactivation during language processing has been replicated using different experimental approaches (Bleich-Cohen et al, 2012; Horn et al, 2012; Li et al, 2007).

There is striking convergence of findings from animal models and behavioral, neurophysiological, neuroimaging, and post-mortem studies strongly supporting pathophysiological models of schizophrenia that involve dysfunction of glutamatergic circuits in the lateral temporal lobe (Javitt and Sweet, 2015). For example, in post-mortem studies, reduction of glutamatergic synapses has been described in patients. Event-related potential studies in animals and humans have related altered mismatch negativity (MMN) responses to glutamate pathology in the STG (Javitt et al, 1992; Umbricht et al, 2000). In schizophrenia patients, there is perturbed hemispheric asymmetry in MMN generation in the STG (Kircher et al, 2004b). Glutamatergic neurotransmission in the lateral temporal lobes has been linked to aberrant auditory sensory processing (P50, MMN), hallucinations, and formal thought disorder (Javitt, 2015). Combining fMRI and NMDAR blockade, either with $R$ - or- $S$-ketamine in healthy volunteers (Abel et al, 2003; Daumann et al, 2009; Deakin et al, 2008; Fu et al, 2005; Honey et al, 2004, 2005; Musso et al, 2011; Nagels et al, 2011, 2012; Vernaleken et al, 2013), altered lateral, temporal, and frontal neural activations were found during overt verbal fluency tasks (Fu et al, 2005; Nagels et al, 2011, 2012). These fMRI results during ketamine challenge resemble neural dysfunctions observed in schizophrenia patients during verbal fluency tasks.

In summary, structural and functional changes in the lateral temporal lobes in schizophrenia have been related to FTD. Pathology of the glutamate system in the STG language areas has been demonstrated in post-mortem and eventrelated potential (ERP) studies. Positive and negative FTD can be elicited in healthy subjects by NMDA blockade with ketamine. During continuous speech production, a right lateral temporal activation has been found in schizophrenia, as opposed to left in healthy subjects. It is yet unclear whether pathology of the glutamate system is causing the perturbed hemispheric activation during speech production in schizophrenia patients with FTD. Therefore, the aim of the current study was to link experimentally induced NMDAR blockade in healthy volunteers to neural activation during an overt, natural speech production fMRI paradigm. The experimental setup and data analysis of the current study is highly analogous to the one used in our previous study, where patients with schizophrenia and prominent FTD were compared with healthy subjects (Kircher et al, 2002a). In this previous patient study we found a correlation between the number of words spoken and activation in the right STG in schizophrenia and the left STG in healthy subjects. In this current study, we hypothesize a similar pattern in healthy subjects, ie, $S$-ketamine as compared with placebo leads to the recruitment of the right lateral temporal cortex during continuous speech production.

\section{MATERIALS AND METHODS}

\section{Subjects}

Fifteen healthy, right-handed (Edinburgh Inventory of Handedness, (Oldfield, 1971)), native German-speaking male volunteers (mean age 27.0 (SD 3.6) years) participated in the study. Subjects with a history of substance abuse or general MRI incompatibility (eg, metal implants) were excluded. Participants were free of medical or neurological diseases. Informed written consent was obtained from all participants. The local ethics committee at RWTH Aachen University approved the study.

\section{Task and Stimuli}

Subjects were required to overtly describe 7 Thematic Apperception Test (TAT) pictures (Murray, 1943) for 3 min each while brain responses were acquired with fMRI. Participants were given a standard set of instructions about the experimental task, asking them to speak about whatever came to their mind on viewing the TAT pictures (see

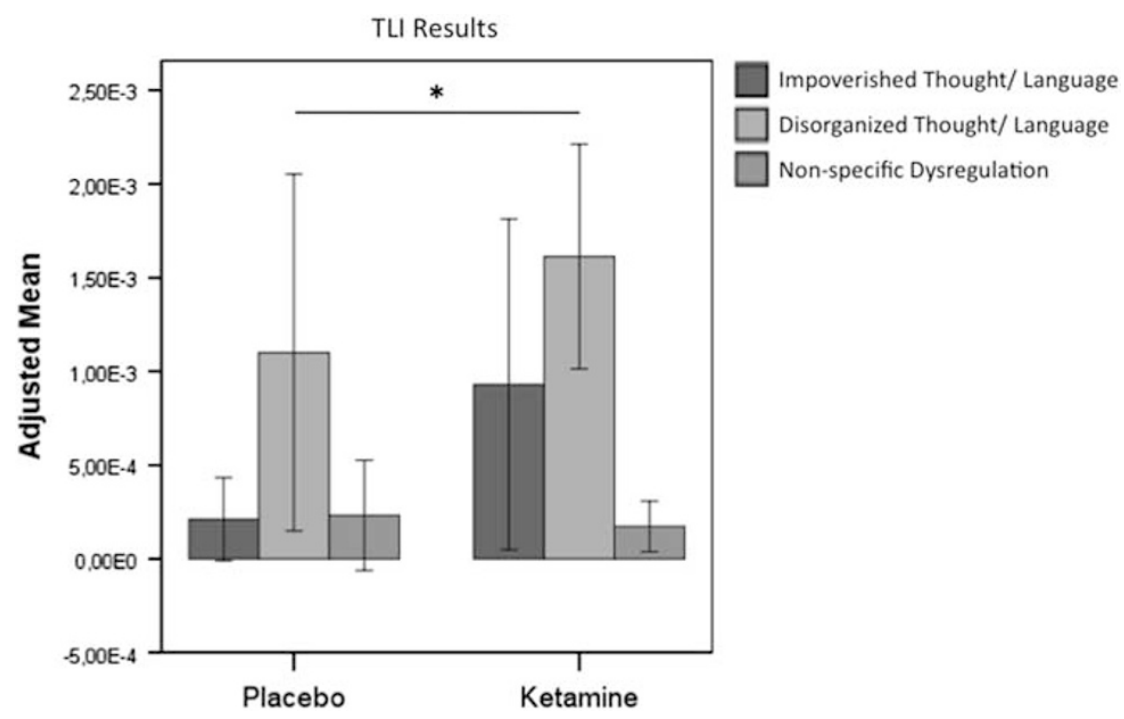

Figure I TLI factor scores for the placebo vs ketamine session. Factor scores on the $y$-axes are adjusted for the number of spoken words during the session (ie, TLI factor value/words). Error bars indicate SD. 
methodological approach by (Kircher et al, 2000)). They were instructed to start speaking as soon as the picture appeared on the screen. The speech production was initiated in a naturalistic manner. There was no prompting if participants paused or stopped talking. In order to avoid sequence effects, 7 from the set of 14 pictures were randomly chosen for the presentation in the scanner (MRI-compatible video goggles: VisuaStim XGA, Resonance Technology, CA, http://www.mrivideo.com/). Subsequently, the 7 TAT pictures were presented at the center of the screen, one at a time for $180 \mathrm{~s}$ (see Figure 1), alternating with a low-level baseline (fixation cross, presented for $30 \mathrm{~s}$ ). Verbal reports were audiotaped with MRI-compatible headset microphone (funcLab, Resonance Technology). All participants wore headphones as well as ear protectors, still allowing them to hear themselves speak. Presentation of stimuli was controlled by a computer using the Presentation 11.0 software package (Neurobehavioral Systems, Albany, CA, http://www.neurobs. $\mathrm{com} /$ ). In order to ensure optimal visual acuity, fMRIcompatible glasses were fixed to the video goggles, if necessary.

Before scanning, all subjects performed two test trials with training pictures that were not used in the fMRI study. A similar experimental task and procedure has been used successfully in a series of previous fMRI studies of ours (see Kircher et al, 2000, 2001b, 2002b, 2004a, 2005).

\section{$S$-Ketamine and Placebo Infusions}

A within-subject, placebo-controlled, counterbalanced design was used. In the ketamine experiment, each subject intravenously (i.v.) first received a bolus of $8 \mathrm{mg} S$-ketamine from a $0.5 \mathrm{mg} / \mathrm{ml}$ solution of $S$-ketamine in $0.9 \% \mathrm{NaCl}$, or a pure saline bolus for the placebo session respectively. This initial bolus was followed by $5 \mathrm{~min}$ with no further $S$ ketamine/placebo supply. Then, the continuous subanesthetic i.v. infusion of $0.01 \mathrm{mg} / \mathrm{kg} / \mathrm{min} S$-ketamine (using a $0.25 \mathrm{mg} / \mathrm{ml}$ solution of $S$-ketamine in $0.9 \% \mathrm{NaCl}$ ) or pure $0.9 \% \mathrm{NaCl}$ for the placebo session was administered using an infusion pump to maintain the initially induced psychopathological symptoms. The duration of the infusion lasted $\sim 1 \mathrm{~h}$. All participants remained in the research facility under medical supervision for at least $2 \mathrm{~h}$ after the termination of the $S$-ketamine infusion.

Ketamine has hitherto been available as a racemic mixture of the two enantiomers $(S)$ and $(R)$ ketamine. The general pharmacological profile of $(S)$-ketamine is largely similar to the racemate. The analgesic and anesthetic potency of $(S)$ ketamine is $\sim 3-5$ times higher than that of the $(R)$-form and twice as high as that of the racemate. To achieve similar effects, a reduction of half the dose is possible with $(S)$ ketamine compared with the racemate. Moreover, $(S)$ ketamine is eliminated more quickly and is therefore more controllable overall. The pure $S$-enantiomer of ketamine displays, at subanesthetic doses, a $4 \pm 5$ times higher affinity for the NMDA receptor than $R$-ketamine. Based on the literature, personal exchange with Franz Vollenweider, Zurich (Vollenweider, 1998; Vollenweider and Geyer, 2001; Vollenweider and Kometer, 2010; Vollenweider et al, 1997a, b; Vollenweider et al, 2000), and own pilot experiments before our scanning study, we used $S$-ketamine in a dose of $0.01 \mathrm{mg} / \mathrm{kg} / \mathrm{min}$ i.v. that corresponds to $0.016 \mathrm{mg} / \mathrm{kg} / \mathrm{min}$ i.v.
$R$-/S-ketamine in terms of eliciting similar psychosis like symptoms (Vollenweider et al, 1997a). The aim was to elicit psychopathological effects, but not sedating people. Moreover, side effects such as nausea and dizziness were not reported at this dosing used in previous works (Nagels et al, 2011,2012 ). A dose of $0.01 \mathrm{mg} / \mathrm{kg} / \mathrm{min}$ i.v. (R)-ketamine does not elicit psychotic symptoms (Vollenweider et al, 1997a). As we were primarily interested in the effects of acute NMDAR blockade in inducing schizophrenia-like symptoms at the speech level and associated brain activation patterns, $S$ ketamine administration in the above dosing appeared to be most reasonable.

\section{Speech Recordings}

Subjects were instructed to speak loud and clearly and to start talking as soon as the stimulus picture appeared. The cable from the microphone as well as the cable from the MR trigger box was plugged into an audio splitter, from which one cable led to the intercom and one to the line-in port of an external sound card attached to a Siemens notebook used for digital recording. All verbal responses were filtered offline (Adobe Audition 3, Adobe Systems Software Ireland, http:// www.adobe.com/) and transcribed. Literal transcripts of these responses were used for quantitative analyses of the participants' overt responses, yielding information about what was said and how many words were produced. For the fMRI data analysis, the seven $180 \mathrm{~s}$ speech production blocks were broken down into $20 \mathrm{~s}$ epochs, resulting in a total number of sixty-three $20 \mathrm{~s}$ blocks for each subject. For each of the $20 \mathrm{~s}$ epochs the number of spoken words was determined and used for the parametric modulation analyses (similar to Kircher et al, 2000).

Psychopathological symptom assessment. The Thought and Language Index (TLI) (Liddle et al, 2002) was used to assess FTD symptoms in the verbatim transcripts. This procedure has been developed and validated to evaluate FTD symptomatology in response to stimulus images in an fMRI environment (Kircher et al, 2002a). A trained, experienced, and blinded clinician (AO) coded each TLI symptom and its severity on the transcripts. As suggested by Liddle et al (2002), a three-dimensional factorial FTD structure was calculated encompassing (1) Impoverished Thought/Language, (2) Disorganized Thought/Language, and (3) Nonspecific Dysregulation (Liddle et al, 2002). Individual factor scores were adjusted for the number of words produced.

\section{fMRI Image Acquisition}

All scanning was performed on a $3 \mathrm{~T}$ scanner (3-T Philips Achieva) using standard gradients and a circular polarized phase array head coil. Participants lay in supine position while head movement was limited by foam padding within the head coil. For each participant, a series of 720 EPI-scans lasting $24 \mathrm{~min}$ was acquired. The EPI scanning technique was adapted to minimize artifacts associated with overt speech production. Scans covered the whole brain parallel to the AC/PC line with the following parameters: number of slices (NS), 34; slice thickness (ST), $4 \mathrm{~mm}$; interslice gap (IG), $1 \mathrm{~mm}$; matrix size (MS), $64 \times 64$; field of view (FOV), $230 \mathrm{~mm}$; echo time (TE), $29 \mathrm{~ms}$; repetition time (TR), $2.0 \mathrm{~s}$. 


\section{fMRI Data Analysis}

MR images were analyzed using Statistical Parametric Mapping software (SPM5; www.fil.ion.ucl.ac.uk) implemented in MATLAB 7.0 (Mathworks, Sherborn, MA). All images were realigned to the first image to correct for head movement. After realignment volumes were normalized into standard stereotaxic anatomical MNI space by using the transformation matrix calculated from the first EPI scan of each subject and the EPI template. Afterwards, the normalized data with a resliced voxel size of $2 \times 2 \times 2 \mathrm{~mm}$ were smoothed with an $8 \mathrm{~mm}$ FWHM isotropic Gaussian kernel to accommodate intersubject variation in brain anatomy.

Before starting the first-level analysis, a visual inspection of the movement parameters ( $x, y, z$, roll, pitch, jaw) was performed; movement did not exceed $6 \mathrm{~mm}$. Moreover, no 'spiking' phenomena were observed. In a further step, movement parameters (realignment data) were individually partialized out in the SPM first-level analysis, as these were included as multiple regressors in the design matrix. In this respect, the effect of speech production on movement did not have an impact on the reported imaging findings.

\section{Hemodynamic Response and Correspondence with Behavior}

The models for the placebo and S-ketamine experiments included each a regressor modeling the hemodynamic responses to word production and a parametric modulator. The latter was done to additionally weight each $20 \mathrm{~s}$ epoch with the corresponding word count. The effect of the parametric weights on word production were computed and analyzed at the group level.

The analysis of behavioral data has shown that $20 \mathrm{~s}$ epochs yield an optimal pattern of high variance and multiple maxima and minima needed for subsequent image analysis (Kircher et al, 2000). On the second-level analysis, onesample $t$-tests were performed to examine differences between conditions. In order to test whether speech production during $S$-ketamine $v s$ placebo was associated with the temporal lobe, we performed a region of interest (ROI) analysis based on the coordinates reported in an earlier study of our group (Kircher et al, 2002a, b), where patients with schizophrenia and healthy subjects were compared during a similar task, RH ROIs: STG $(x=46$, $y=-25, z=9)$, MTG $(x=58, y=-25, z=4)$, and ITG $(x=40, y=-6, z=-24)$ (coordinates refer to peak voxel
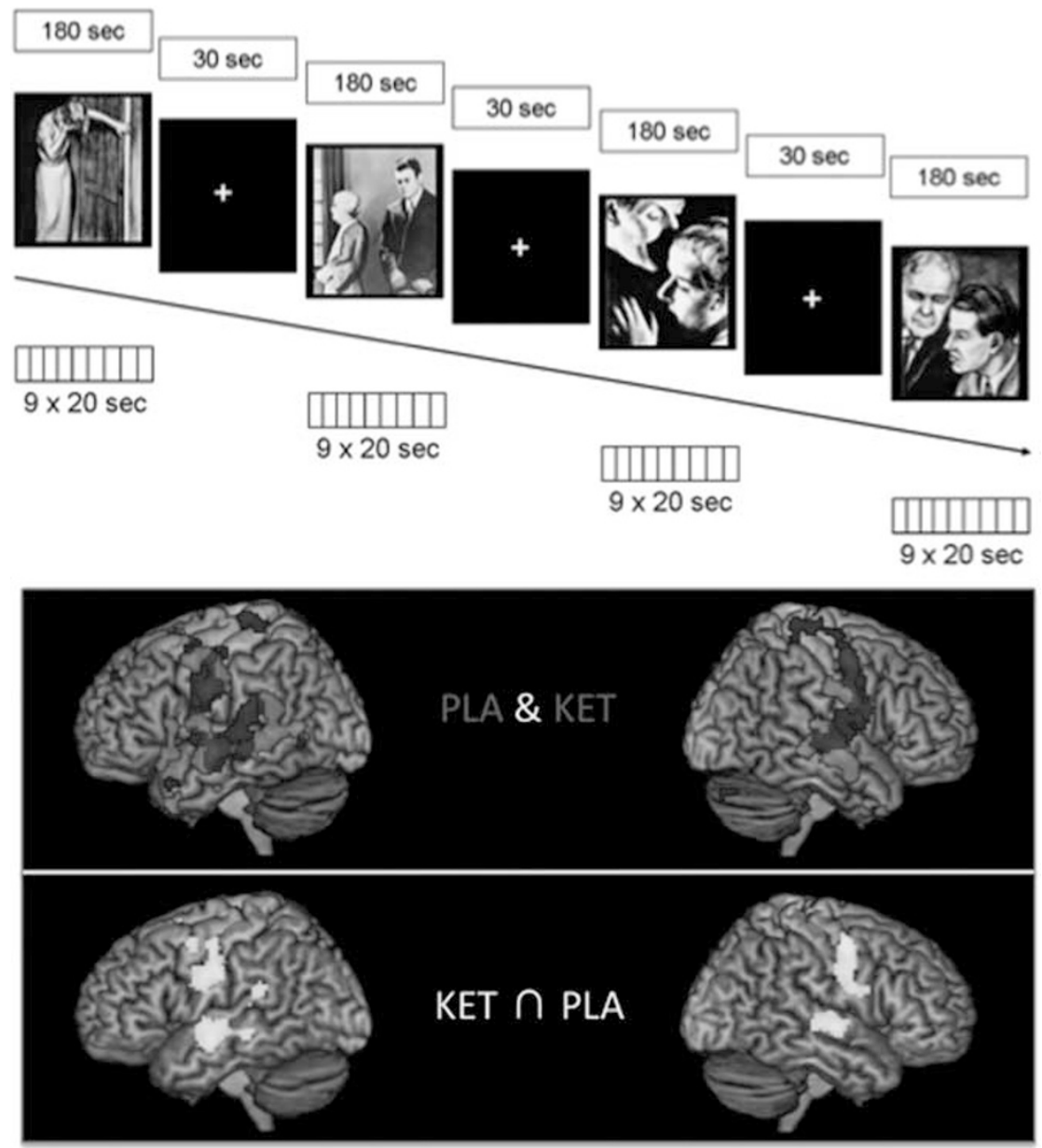

Figure 2 Top, experimental setup: Speech production (each TAT picture: $180 \mathrm{~s}$ ) and baseline resting (fixation cross: 30 s) phase. For the analysis each speech production phase (7 pictures $\times 180 \mathrm{~s}=1.260 \mathrm{~s}$ ) was subdivided into $20 \mathrm{~s}$ blocks (63 in total) and correlated with the BOLD effect. Middle panel: Overlay image of brain regions activated in connection with a higher amount of speech production during placebo $(P L A=b l u e)$ and ketamine $(K E T=$ green) $\left(p<0.00 \mathrm{I}, K_{\mathrm{e}}=10 \mathrm{v}\right)$. Lower panel: Brain regions activated in both sessions together (PLA $\cap$ KET conjunction analysis, $p<0.00 \mathrm{I}, K_{\mathrm{e}}=10 \mathrm{v}$ ). A full color version of this figure is available at the Neuropsychopharmacology journal online. 
activations). Furthermore, an exploratory whole-brain analysis was run to detect brain regions being associated with $S$ ketamine-induced neural responses. Brain activations were plotted on the anatomical SPM template.

\section{RESULTS}

\section{Behavioral Results}

Psychopathology. S-ketamine elicited psychopathological symptoms, measured outside the scanner, similar to those generally seen in patients with schizophrenia (PANSS pos. mean 11.93, SD 1.75; PANSS neg. 18.20, SD 5.13; PANSS gen. 22.67, SD 2.53; PANSS total 52.80, SD 7.10) (Kay et al, 1987).

Word count. Significantly more words were produced within the $20 \mathrm{~s}$ fMRI epochs during the placebo session (mean per $20 \mathrm{~s}=36.40 ; \mathrm{SD}=11.13$ ) as compared with the $S$ ketamine session $($ mean $=28.98 ; \mathrm{SD}=11.97)$ (Wilcoxon test: $z=-2.56 ; p=0.01$ ). No effect of order (conditions: ketamine or placebo) on the number of words produced was found (Pearson's $\chi^{2}: p=0.41$ ).

TLI ratings. During fMRI scanning, the TLI factor Disorganized thought/language was significantly increased in the $S$-ketamine $v s$ placebo session $(z=1.93, p=0.05$, Figure 1). A trend to significance was found for the factor score Impoverished thought/language $(z=1.86, p=0.06$, Figure 1). Nonspecific Dysregulation did not differ between the sessions $(z=0.15, p=0.88$, Figure 1$)$.

Imaging results. Rate of articulation: whole-brain conjunction analysis across both sessions. Brain regions where BOLD responses correlated positively with the amount of speech produced during both sessions (KET and PLA, conjunction analysis) comprised the bilateral precentral and postcentral as well as the bilateral superior temporal regions (see Figure 2). The cluster of activation in the left temporal lobe encompassed the superior temporal gyrus, the middle temporal gyrus, as well as the inferior part of the parietal lobe. The right-hemispheric cluster of activation extended from the precentral region to the right superior temporal gyrus. Moreover, bi-hemispheric positive correlations in the cerebellum as well as in the left middle frontal gyrus (SMA) were detected.

\section{ROI Analysis}

The rate of articulation was correlated with the BOLD effect in the PLA and KET sessions. In a next step, differences between sessions were calculated. The ROI analyses revealed two clusters of statistical differences, one in the right inferior temporal gyrus (activation peak: $x=52, y=6, z=-36$, $t=5.15, K_{\mathrm{e}}=28$ ) and the other in the right middle temporal gyrus extending to the superior temporal gyrus (activation peak: $\left.x=58, y=-34, z=-4, t=4.57, K_{\mathrm{e}}=9\right)$.

In addition, no order effects of condition (ketamine or placebo) were detected on the level of brain activation (MTG: $p=0.36$, ITG: $p=0.41$, Hippocampus: $p=0.45$ ).

\section{Whole-Brain Analysis}

In the S-ketamine $v s$ placebo session, higher amount articulation revealed enhanced neural responses in the right inferior and middle temporal gyri, the left superior occipital gyrus, the left calcarine gyrus, as well as the left hippocampal and the right inferior parietal region. The cluster in the right inferior temporal gyrus extended to the medial temporal region. The inverse contrast (PLA $>$ KET) revealed activations in the bilateral cerebellum and in the right angular gyrus (Table 1 and Figure 3).

\section{Association between FTD Symptoms and Potential Sedation}

It could be argued that the subjects spoke thought disordered because of 'sleepiness', a potentially sedative effect of ketamine, rather than 'psychosis' induced by NMDA blockade. The hypothetical point implied would be that FTD symptoms induced by 'sleepiness' would involve a different neural network than the identical symptoms induced by 'model psychosis'.

To address this point, we recalculated the behavioral data with the condition (ketamine $v s$ placebo) as between-subjects factor and the PANSS item for motor retardation-motor retardation is a good proxy for sedative effects-as a covariate. The number of words still significantly differs

Table I Whole-Brain Analysis: Correlations for Placebo Vs Ketamine

\begin{tabular}{|c|c|c|c|c|c|c|c|}
\hline \multirow[b]{2}{*}{ Contrast } & \multirow[b]{2}{*}{ Anatomical region } & \multirow[b]{2}{*}{$\mathbf{H}$} & \multicolumn{3}{|c|}{ MNI coordinates } & \multirow[b]{2}{*}{$t$-value } & \multirow[b]{2}{*}{ Cluster size } \\
\hline & & & $x$ & $y$ & $z$ & & \\
\hline & Angular gyrus & $\mathrm{R}$ & 52 & -64 & 42 & 4.10 & 8 \\
\hline \multirow[t]{4}{*}{$K E T>P L A$} & Hippocampus & $L$ & -30 & -14 & -10 & 6.04 & 19 \\
\hline & Superior occipital gyrus & $L$ & -14 & -98 & 24 & 4.57 & 18 \\
\hline & Superior parietal lobe & $\mathrm{R}$ & 38 & -30 & 36 & 4.03 & 5 \\
\hline & Calcarine gyrus & $\mathrm{L}$ & -6 & -102 & -8 & 3.79 & 6 \\
\hline
\end{tabular}



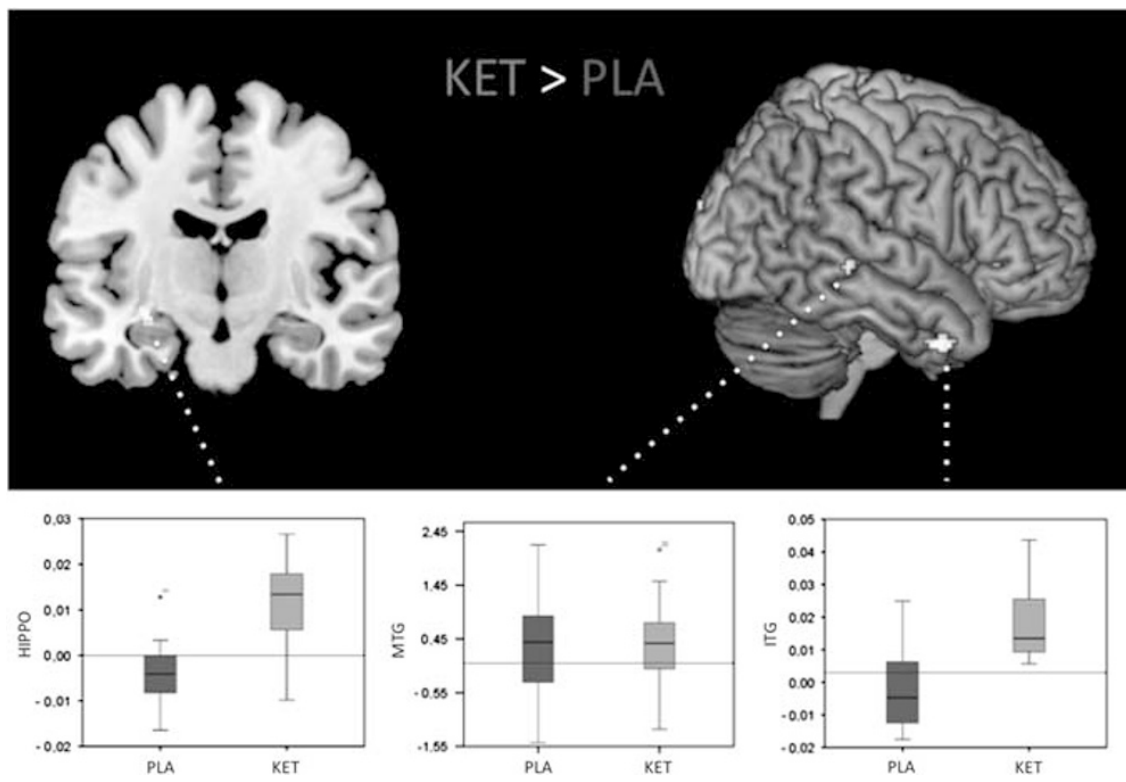

Figure 3 Whole-brain analysis: regions correlating with the rate of articulation for the differential contrast KET (green) $>$ PLA (blue) ( $p<0.00 \mathrm{I}$, for illustrative purposes $K_{e}=5$ voxels). HIPPO, hippocampus; MTG, middle temporal gyrus; ITG, inferior temporal gyrus. A full color version of this figure is available at the Neuropsychopharmacology joumal online.

between conditions, indicating that motor retardation did not directly influence the behavioral data.

Regarding neural activations, post hoc correlation analysis was performed between formal thought disorder symptoms recorded during the scanning procedure ('TLI'; Liddle et al, 2002) and the BOLD signal changes (S-ketamine vs placebo). For this purpose, the TLI scores for the three different formal thought disorder dimensions ('disorganization,' 'dysregulation,' and 'impoverishment') were related to BOLD signal changes in the MTG, ITG, and hippocampus (see Figure 4).

TLI factor scores were found to be differentially associated with neural responses in the ITG, MTG, and hippocampus. Thus, a positive correlation was found for the 'Disorganization' dimension (Spearman's Rho $=0.43, p=0.05$ ) as well as for the 'Dysregulation' factor (Spearman's Rho $=0.43$, $p=0.05)$ and BOLD signal changes in the MTG. Symptoms of 'Impoverishment' were negatively related to neural responses in the MTG (Spearman's Rho $=-0.40, p=0.07$ ).

With regard to the relationship between right ITG activations and TLI FTD dimensions, no significant correlations were found ('Impoverishment': Spearman's Rho= $-0.05, p=0.44$; 'Disorganization': Spearman's Rho $=-0.09$, $p=0.38$; 'Dysregulation': Spearman's Rho $=-0.07, p=0.41$ ). For the association between BOLD signal changes in the hippocampus and TLI scores, the factors were not associated to BOLD signal changes in the hippocampal region ('Disorganization': Spearman's Rho $=-0.20, p=0.24$; 'Dysregulation': Spearman's Rho $=0.20, p=0.24$; 'Impoverishment' Spearman's Rho $=-0.37, p=0.09$ ) (Figure 4).

\section{DISCUSSION}

We investigated the involvement of the glutamate system in thought and language processes. S-ketamine infusion vs placebo was administrated during fMRI in healthy subjects to induce FTD, similar to those observed in patients with schizophrenia (Adler et al, 1999). Subjects were scanned with

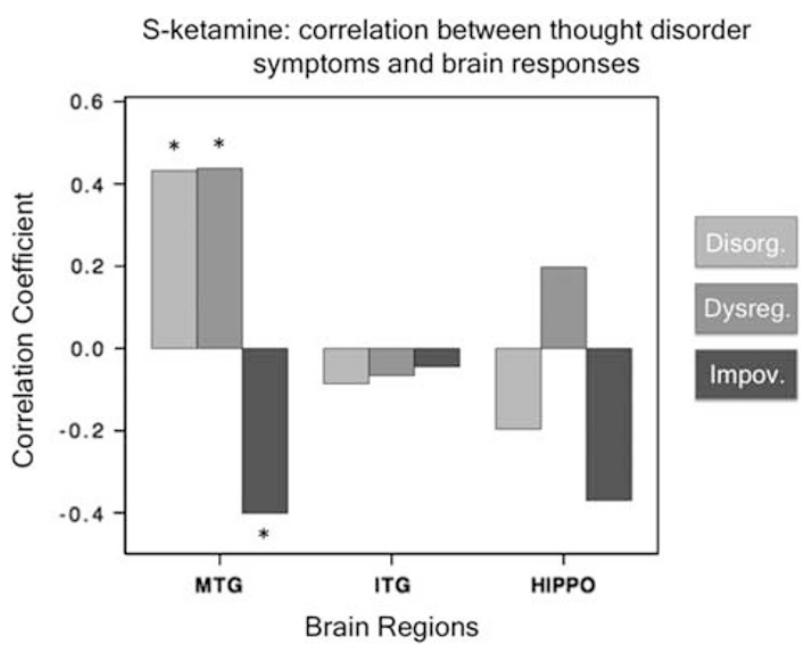

Figure 4 Correlation between BOLD signal changes (ketamine vs placebo) and TLI parameters under S-ketamine (orange $=$ disorganization, red $=$ dysregulation, and blue $=$ impoverishment; $* p \leq 0.05)$. A full color version of this figure is available at the Neuropsychopharmacology journal online.

fMRI while they freely spoke about TAT pictures. The amount of speech production during $20 \mathrm{~s}$ epochs was correlated with the BOLD effect. The amount of articulation during S-ketamine vs placebo was associated with neural responses in right-hemispheric inferior and middle temporal gyri, consistent across both ROI and whole-brain analyses. The same experimental approach was used in a previous study involving healthy subjects and FTD patients with schizophrenia, without infusions (Kircher et al, 2002a, b). The current results during NMDAR blockade in healthy subjects are strikingly similar to those observed in the schizophrenia study (Kircher et al, 2002a, b). We conclude that alterations on the synaptic level in the glutamate system 
of the lateral temporal lobes lead to reversed brain activation $(\mathrm{R}>\mathrm{L})$ during continuous, natural speech, and consequently to (positive) formal thought disorders in patients with schizophrenia.

\section{Behavioral Results}

In line with previous results, acute NMDAR blockade induced psychosis-like symptoms, particularly positive and negative FTD, as measured with the TLI (Fu et al, 2005; Nagels et al, 2011, 2012; Vollenweider et al, 2000). Under Sketamine, healthy subjects produced significantly less words compared with placebo. This is also in line with the previous study of ours in schizophrenia patients who were selected for exhibiting prominent positive FTD and also produced fewer words than the healthy controls (Kircher et al, 2002a, b). The results of the current study therefore validate the $S$-ketamine model of psychosis for the psychopathological effects in the speech production system.

\section{Neural Results and Glutamate System}

On a neural level, the rate of articulation in the $S$-ketamine experiment ( $v s$ placebo) correlated with BOLD responses in right-hemispheric inferior and middle temporal gyri, consistent across both ROI and whole-brain analyses. The current result for $S$-ketamine is thus strikingly similar to the one found in schizophrenia patients with positive FTD from our earlier work, where we used an almost identical experimental setup and analysis. Enhanced right temporal lobe recruitment or diminished functional lateralization of language is a replicated finding in schizophrenia patients (Bleich-Cohen et al, 2009), but its etiology was elusive until now.

There is evidence from several levels of investigation for the involvement of the glutamate system in lateral temporal lobe pathology in schizophrenia (for review see Javitt, 2015). Immunocytochemical post-mortem studies of the glutamate system have investigated the integrity of supragranular pyramidal cells within the STG in schizophrenia. They found a reduction in dendritic spine density in deep layer 3 of the primary auditory cortex in individuals with schizophrenia (Sweet et al, 2009). Importantly, the reductions in spine density did not result from a loss of pyramidal neurons that were unchanged in number (Dorph-Petersen et al, 2009), but instead from a reduction in the number of spines per neuron, potentially leading to a reduction in neuronal connectivity. The reductions in spine number could also not be attributed to long-term antipsychotic treatment. A targeted proteomic analysis of 155 synaptic proteins in the primary auditory cortex tissue found that schizophrenia is associated with the altered expression of various glutamatergic signaling proteins (MacDonald et al, 2015). The two most significantly reduced proteins were GluR3 and GluR4, the calcium-permeable subunits of AMPA receptors. NMDAR protein levels were found to be unchanged. The expression levels of VGluT1 and VGluT2 within glutamatergic boutons are preserved in this brain region, suggesting that the packing of glutamate into vesicles and response upon vesicle release would be intact. Reductions in presynaptic proteins may affect postsynaptic pyramidal neuron structural integrity. Because deafferentation of glutamatergic projections is known to lead to decreased spine density (Cheng et al, 1997; McKinney et al, 1999), reductions in synaptophysin within glutamate boutons in layer 3 of the cortex might further contribute to functional glutamatergic deafferentation in the temporal cortex of individuals with schizophrenia (McKinney et al, 1999). Many of the glutamatergic alterations in schizophrenia are not unique to the primary auditory cortex. Alterations in the morphology of pyramidal neurons, including dendritic spine density reductions, have been reported in other cortical regions, including the auditory association cortex, temporal association cortex, prefrontal cortex, and hippocampal formation ( $\mathrm{Hu}$ et al, 2015).

Further indirect evidence for an alteration of the glutamatergic system in the auditory cortex and lateral temporal lobes in patients comes from acoustic ERP studies. In particular, $\mathrm{MMN}$ is an electrophysiological correlate of sensory memory representation and can be observed even under distracted sessions. This preattentive response is generated at the level of the auditory cortex (Kircher et al, 2004b) and is likely to reflect NMDA channel current influx in cortical layers 2 and 3 as found in extracellular recordings in the monkey (Javitt et al, 1992). In humans, a diminished mismatch negativity response following the administration of ketamine has been described (Umbricht et al, 2000), consistent with NMDA/glutamate involvement in MMN generation. These effects of NMDAR antagonists on MMN generation have been replicated in multiple human, monkey, and rodent studies (Javitt, 2015). The MMN response to duration deviants, usually recorded with EEG, is consistently reduced in patients with schizophrenia (Todd et al, 2013). In a combined fMRI and MEG study on MMN, an altered hemispheric lateralization of neuromagnetic mismatch fields and corresponding BOLD effects in the STG in schizophrenia patients has been demonstrated (Kircher et al, 2004b). In summary, there is multiple evidence for an altered glutamatergic transmission in the lateral temporal lobes of patients with schizophrenia (Javitt, 2015).

\section{Temporal Lobes and FTD in Schizophrenia}

Early imaging studies using either continuous production or sentence completion tasks found a pronounced activation of the right hemispheric temporal region in patients with FTD schizophrenia vs healthy controls (Kircher et al, 2001a, 2002a). Positive as well as negative FTD have been consistently related to dysactivation in the lateral posterior temporal gyri (Horn et al, 2009, 2010, 2012; Kircher et al, 2001b; McGuire et al, 1998). The majority of structural brain imaging studies found cortical thinning or hypogyrification (Palaniyappan et al, 2013) in the STG/MTG/ITG and planum temporale (Ratnanather et al, 2013). This structural finding is most prominent in patients with FTD and was also found in genetically high-risk subjects for schizophrenia ( $\mathrm{Li}$ et al, 2007). Anatomical connectivity disruptions in the lefthemispheric language regions were found by diffusion tensor imaging (DTI) studies (Jeong et al, 2009), Asami et al, 2014). Furthermore, reduced volume of the corpus callosum in schizophrenia might also be responsible for impaired interhemispheric transfer. Thus, there is overwhelming evidence for aberrations in the lateral temporal lobes in schizophrenia on a gray and white matter structural as well 
as functional level, particularly in those patients with prominent positive FTD.

In order to test a possible sedation effect on our behavioral results, we recalculated the behavioral data with the condition (ketamine vs placebo) as between-subjects factor and the PANSS item for motor retardation-a good proxy for sedative effects - as a covariate. The number of words still significantly differed between conditions indicating that motor retardation did not directly influence these data. Moreover, in our previous study in patients with schizophrenia (Kircher et al, 2001a, b) patients also spoke fewer words as compared with the control subjects, but they were not sedated by medication.

To rule out ketamine-induced sedative effects on the differential activation in the MTG, we correlated the obtained TLI factor scores with BOLD signal changes (ketamine vs placebo). This procedure revealed that in particular the "key region'-the right $\mathrm{MTG}$ - shows a positive correlation with positive FTD factors of the TLI, that is, 'Disorganization' and 'Dysregulation', in line with our hypothesis.

Taken together with the results from our current study, it is evident that in patients with schizophrenia hemispheric asymmetry is altered on structural and functional levels with a major involvement of the glutamate system, leading to a recruitment of the $\mathrm{RH}$ temporal cortex during speech production that results phenomenologically in FTD.

\section{Left Vs Right Hemispheric Speech Production}

What are the putative effects of right temporal recruitment during speech production? The right lateral temporal lobe is needed for the understanding of longer phrases. However, its recruitment during production results in imprecise semantic output during speech articulation (Chiarello et al, 1990; Faust and Chiarello, 1998). In our placebo session, continuous speech production primarily involved the left STG, where narrow semantic fields facilitate precise word retrieval and the maintenance of a focused, logical trail of thought. In the $S$-ketamine session, continuous speech was associated with signal changes in the right STG, where diffuse semantic fields activate several semantically and phonologically related lexical items at the same time. Thus, lexical retrieval in the $\mathrm{RH}$ during $S$-ketamine may be relatively imprecise, increasing the probability that words only related to the intended concept (as opposed to the intended concept/word per se) are retrieved. This imprecision contributes to the articulation of positive FTD.

\section{Conjunction Analysis}

In the S-ketamine and placebo experimental sessions, the amount of speech production was associated with enhanced BOLD responses in the 'language network' encompassing cortical motor regions (bilateral precentral and postcentral gyri) as well as bilateral temporal areas. A similar result has previously been reported in our analogous study (Kircher et al, 2000). The STG and MTG have been related to depth of semantic processing such that the STG-as part of the dorsal (articulatory) language stream (Hickok and Poeppel, 2007) -is not simply a passive store of lexical-semantic information, but is involved in active retrieval (Ruff et al, 2008; Wilson et al, 2009). Furthermore, motor planning, execution, and monitoring of the verbal output have also been related to STG and MTG activation (Kircher et al, 2000, 2002a).

\section{Limitations}

We acknowledge that the dopamine, GABA, and glutamate system are deeply interconnected, are all relevant for schizophrenia, and may be related to FTD symptomatology. It is beyond the scope of one study though to investigate their intricate relationships, their connection to psychopathology, and brain activation at the same time.

It is not yet entirely clear which cognitive processes are related to the rate of articulation during continuous language production. As mentioned above, there are several subprocesses involved being closely intertwined such as planning, lexico-semantic retrieval, motor encoding, and execution. Further studies investigating different neurotransmitter systems may want to probe particular subprocesses during speech production.

\section{CONCLUSION}

Our results support the view that a pathological glutamate system is in part responsible for psychotic symptoms, language dysfunctions, and aberrations on the neural level. Neurodevelopmental affects result in biochemical and synaptic alterations of glutamatergic neurons in the lateral temporal lobes (Javitt and Sweet, 2015). This gives rise to cortical thinning, white matter changes, and functional alterations in these areas, detectable in vivo with MRI techniques. Both the resulting hemispheric dysactivation within the temporal language network during speech production and the (additional) recruitment of the right temporal lobe result in the formation of FTD symptoms. Future studies should focus on investigating the causes of glutamatergic dysfunction and the consequences for the pathogenesis of schizophrenia. A deeper understanding of its neurophysiological implications may open new windows for the development of novel NMDARbased treatment strategies.

\section{FUNDING AND DISCLOSURE}

The authors declare no conflict of interest.

\section{ACKNOWLEDGMENTS}

We thank Karsten Stragies for his support with respect to the recordings and transcriptions of the audio files. We thank Franz $\mathrm{X}$ Vollenweider for his assistance and help regarding the $S$ ketamine dosing. TK has received unlimited educational grants for symposia from Servier Deutschland $\mathrm{GmbH}$, Actelion Pharmaceuticals Deutschland $\mathrm{GmbH}$, Janssen-Cilag $\mathrm{GmbH}$, Lundbeck GmbH, Trommsdorff GmbH \& Co. KG Arzneimittel, Lilly Deutschland $\mathrm{GmbH}$, and Aristo Pharma $\mathrm{GmbH}$.

\section{REFERENCES}

Abel KM, Allin MP, Kucharska-Pietura K, Andrew C, Williams S, David AS et al (2003). Ketamine and fMRI BOLD signal: distinguishing between effects mediated by change in blood flow versus change in cognitive state. Hum Brain Mapp 18: 135-145. 
Abi-Saab WM, D'Souza DC, Moghaddam B, Krystal JH (1998). The NMDA antagonist model for schizophrenia: promise and pitfalls. Pharmacopsychiatry 31(Suppl 2): 104-109.

Adler CM, Goldberg TE, Malhotra AK, Pickar D, Breier A (1998). Effects of ketamine on thought disorder, working memory, and semantic memory in healthy volunteers. Biol Psychiatry 43: 811-816.

Adler CM, Malhotra AK, Elman I, Goldberg T, Egan M, Pickar D et al (1999). Comparison of ketamine-induced thought disorder in healthy volunteers and thought disorder in schizophrenia. Am J Psychiatry 156: 1646-1649.

Asami T, Lee SH, Bouix S, Rathi Y, Whitford TJ, Niznikiewicz M et al (2014). Cerebral white matter abnormalities and their associations with negative but not positive symptoms of schizophrenia. Psychiatry Research 222: 52-59.

Bleich-Cohen M, Hendler T, Kotler M, Strous RD (2009). Reduced language lateralization in first-episode schizophrenia: an fMRI index of functional asymmetry. Psychiatry Res 171: 82-93.

Bleich-Cohen M, Sharon H, Weizman R, Poyurovsky M, Faragian S, Hendler T (2012). Diminished language lateralization in schizophrenia corresponds to impaired inter-hemispheric functional connectivity. Schizophr Res 134: 131-136.

Cheng HW, Rafols JA, Goshgarian HG, Anavi Y, Tong J, McNeill TH (1997). Differential spine loss and regrowth of striatal neurons following multiple forms of deafferentation: a Golgi study. Exp Neurol 147: 287-298.

Chiarello C, Burgess C, Richards L, Pollock A (1990). Semantic and associative priming in the cerebral hemispheres: some words do, some words don't ... sometimes, some places. Brain Lang 38: 75-104.

Daumann J, Wagner D, Heekeren K, Neukirch A, Thiel C, Gouzoulis-Mayfrank E (2010). Neuronal correlates of visual and auditory alertness in the DMT and ketamine model of psychosis. J Psychopharmacol 24: 1515-1524.

Deakin JF, Lees J, McKie S, Hallak JE, Williams SR, Dursun SM (2008). Glutamate and the neural basis of the subjective effects of ketamine: a pharmaco-magnetic resonance imaging study. Arch Gen Psychiatry 65: 154-164.

Dorph-Petersen KA, Delevich KM, Marcsisin MJ, Zhang W, Sampson AR, Gundersen HJ et al (2009). Pyramidal neuron number in layer 3 of primary auditory cortex of subjects with schizophrenia. Brain Res 1285: 42-57.

Faust M, Chiarello C (1998). Sentence context and lexical ambiguity resolution by the two hemispheres. Neuropsychologia 36: 827-835.

Fu CH, Abel KM, Allin MP, Gasston D, Costafreda SG, Suckling J et al (2005). Effects of ketamine on prefrontal and striatal regions in an overt verbal fluency task: a functional magnetic resonance imaging study. Psychopharmacology (Berl) 183: 92-102.

Griesinger W (1861). Die Pathologie und Therapie der psychischen Krankheiten 2 ednKrabbe: Stuttgart.

Hickok G, Poeppel D (2007). The cortical organization of speech processing. Nat Rev Neurosci 8: 393-402.

Honey GD, Honey RA, O'Loughlin C, Sharar SR, Kumaran D, Suckling $J$ et al (2005). Ketamine disrupts frontal and hippocampal contribution to encoding and retrieval of episodic memory: an fMRI study. Cereb Cortex 15: 749-759.

Honey RA, Honey GD, O'Loughlin C, Sharar SR, Kumaran D, Bullmore ET et al (2004). Acute ketamine administration alters the brain responses to executive demands in a verbal working memory task: an FMRI study. Neuropsychopharmacology 29: 1203-1214.

Horn H, Federspiel A, Wirth M, Muller TJ, Wiest R, Wang JJ et al (2009). Structural and metabolic changes in language areas linked to formal thought disorder. Br J Psychiatry 194: 130-138.

Horn H, Federspiel A, Wirth M, Muller TJ, Wiest R, Walther S et al (2010). Gray matter volume differences specific to formal thought disorder in schizophrenia. Psychiatry Res 182: 183-186.

Horn H, Jann K, Federspiel A, Walther S, Wiest R, Muller T et al (2012). Semantic network disconnection in formal thought disorder. Neuropsychobiology 66: 14-23.
$\mathrm{Hu}$ W, MacDonald ML, Elswick DE, Sweet RA (2015). The glutamate hypothesis of schizophrenia: evidence from human brain tissue studies. Ann NY Acad Sci 1338: 38-57.

Javitt DC (2015). Neurophysiological models for new treatment development in schizophrenia: early sensory approaches. Ann NY Acad Sci 1344: 92-104.

Javitt DC, Schroeder CE, Steinschneider M, Arezzo JC, Vaughan HG Jr. (1992). Demonstration of mismatch negativity in the monkey. Electroencephalogr Clin Neurophysiol 83: 87-90.

Javitt DC, Sweet RA (2015). Auditory dysfunction in schizophrenia: integrating clinical and basic features. Nat Rev Neurosci 16: 535-550.

Jeong B, Wible CG, Hashimoto R, Kubicki M (2009). Functional and anatomical connectivity abnormalities in left inferior frontal gyrus in schizophrenia. Hum Brain Mapp 30: 4138-4151.

Kantrowitz JT, Javitt DC (2010). N-methyl-d-aspartate (NMDA) receptor dysfunction or dysregulation: the final common pathway on the road to schizophrenia? Brain Res Bull 83: 108-121.

Kay SR, Fiszbein A, Opler LA (1987). The positive and negative syndrome scale (PANSS) for schizophrenia. Schizophr Bull 13: 261-276.

Kircher T, Liddle PF, Brammer MJ, Williams SC, Murray RM, McGuire PK (2002a). Reversed lateralization of temporal activation during speech production in thought disordered patients with schizophrenia. Psychol Med 32: 439-449.

Kircher TT, Brammer MJ, Levelt W, Bartels M, McGuire PK (2004a). Pausing for thought: engagement of left temporal cortex during pauses in speech. Neuroimage 21: 84-90.

Kircher TT, Brammer MJ, Williams SC, McGuire PK (2000). Lexical retrieval during fluent speech production: an fMRI study. Neuroreport 11: 4093-4096.

Kircher T, Bröhl H, Meier F, Engelen J. Formal Thought Disorders (FTD): From Phenomenology to Neurobiology. Lancet Psychiatry, 2018, in press.

Kircher TT, Bulimore ET, Brammer MJ, Williams SC, Broome MR, Murray RM et al (2001a). Differential activation of temporal cortex during sentence completion in schizophrenic patients with and without formal thought disorder. Schizophr Res 50: 27-40.

Kircher TT, Liddle PF, Brammer MJ, Williams SC, Murray RM, McGuire PK (2001b). Neural correlates of formal thought disorder in schizophrenia: preliminary findings from a functional magnetic resonance imaging study. Arch Gen Psychiatry 58: 769-774.

Kircher TT, Liddle PF, Brammer MJ, Williams SC, Murray RM, McGuire PK (2002b). Reversed lateralization of temporal activation during speech production in thought disordered patients with schizophrenia. Psychol Med 32: 439-449.

Kircher TT, Oh TM, Brammer MJ, McGuire PK (2005). Neural correlates of syntax production in schizophrenia. $\mathrm{Br} J$ Psychiatry 186: 209-214.

Kircher TT, Rapp A, Grodd W, Buchkremer G, Weiskopf N, Lutzenberger W et al (2004b). Mismatch negativity responses in schizophrenia: a combined fMRI and whole-head MEG study. Am J Psychiatry 161: 294-304.

Li X, Branch CA, Ardekani BA, Bertisch H, Hicks C, DeLisi LE (2007). fMRI study of language activation in schizophrenia, schizoaffective disorder and in individuals genetically at high risk. Schizophr Res 96: 14-24.

Liddle PF, Ngan ET, Caissie SL, Anderson CM, Bates AT, Quested DJ et al (2002). Thought and Language Index: an instrument for assessing thought and language in schizophrenia. Br J Psychiatry 181: 326-330.

MacDonald ML, Ding Y, Newman J, Hemby S, Penzes P, Lewis DA et al (2015). Altered glutamate protein co-expression network topology linked to spine loss in the auditory cortex of schizophrenia. Biol Psychiatry 77: 959-968.

McGuire PK, Quested DJ, Spence SA, Murray RM, Frith CD, Liddle PF (1998). Pathophysiology of 'positive' thought disorder in schizophrenia. Br J Psychiatry 173: 231-235. 
McKinney RA, Capogna M, Durr R, Gahwiler BH, Thompson SM (1999). Miniature synaptic events maintain dendritic spines via AMPA receptor activation. Nat Neurosci 2: 44-49.

Murray HA (1943). Thematic Apperception Test. Harvard University Press: Cambridge.

Musso F, Brinkmeyer J, Ecker D, London MK, Thieme G, Warbrick $\mathrm{T}$ et al (2011). Ketamine effects on brain function - simultaneous fMRI/EEG during a visual oddball task. Neuroimage 58: 508-525.

Nagels A, Kirner-Veselinovic A, Krach S, Kircher T (2011). Neural correlates of S-ketamine induced psychosis during overt continuous verbal fluency. Neuroimage 54: 1307-1314.

Nagels A, Kirner-Veselinovic A, Wiese R, Paulus FM, Kircher T, Krach S (2012). Effects of ketamine-induced psychopathological symptoms on continuous overt rhyme fluency. Eur Arch Psychiatry Clin Neurosci 262: 403-414.

Oertel-Knochel V, Knochel C, Stablein M, Linden DE (2012). Abnormal functional and structural asymmetry as biomarker for schizophrenia. Curr Top Med Chem 12: 2434-2451.

Oldfield RC (1971). The assessment and analysis of handedness: the Edinburgh inventory. Neuropsychologia 9: 97-113.

Palaniyappan L, Crow TJ, Hough M, Voets NL, Liddle PF, James S et al (2013). Gyrification of Broca's region is anomalously lateralized at onset of schizophrenia in adolescence and regresses at 2 year follow-up. Schizophr Res 147: 39-45.

Ratnanather JT, Poynton CB, Pisano DV, Crocker B, Postell E, Cebron S et al (2013). Morphometry of superior temporal gyrus and planum temporale in schizophrenia and psychotic bipolar disorder. Schizophr Res 150: 476-483.

Ruff I, Blumstein SE, Myers EB, Hutchison E (2008). Recruitment of anterior and posterior structures in lexical-semantic processing: an fMRI study comparing implicit and explicit tasks. Brain Lang 105: 41-49.

Sweet RA, Henteleff RA, Zhang W, Sampson AR, Lewis DA (2009). Reduced dendritic spine density in auditory cortex of subjects with schizophrenia. Neuropsychopharmacology 34: 374-389.
Todd J, Harms L, Schall U, Michie PT (2013). Mismatch negativity: translating the potential. Front Psychiatry 4: 171.

Umbricht D, Schmid L, Koller R, Vollenweider FX, Hell D, Javitt DC (2000). Ketamine-induced deficits in auditory and visual context-dependent processing in healthy volunteers: implications for models of cognitive deficits in schizophrenia. Arch Gen Psychiatry 57: 1139-1147.

Vernaleken I, Klomp M, Moeller O, Raptis M, Nagels A, Rosch F et al (2013). Vulnerability to psychotogenic effects of ketamine is associated with elevated D2/3-receptor availability. Int J Neuropsychopharmacol 16: 745-54.

Vollenweider FX, Vontobel P, Oye I, Hell D, Leenders KL (2000). Effects of (S)-ketamine on striatal dopamine: a [11C]raclopride PET study of a model psychosis in humans. J Psychiatr Res 34: 35-43.

Vollenweider F (1998). Advances and pathophysiological models of hallucinogenic drug actions in humans: a preamble to schizophrenia research. Pharmacopsychiatry 31S2: 92-103.

Vollenweider FX, Geyer MA (2001). A systems model of altered consciousness: integrating natural and drug-induced psychoses. Brain Res Bull 56: 495-507.

Vollenweider FX, Kometer M (2010). The neurobiology of psychedelic drugs: implications for the treatment of mood disorders. Nat Rev Neurosci 11: 642-651.

Vollenweider FX, Leenders KL, Scharfetter C, Antonini A, Maguire P, Missimer J, Angst J (1997a). Metabolic hyperfrontality and psychopathology in the ketamine model of psychosis using positron emission tomography (PET) and [18F]fluorodeoxyglucose (FDG). Eur Neuropsychopharmacol 7: 9-24.

Vollenweider FX, Leenders KL, Oye I, Hell D, Angst J (1997b). Differential psychopathology and patterns of cerebral glucose utilisation produced by (S)- and (R)-ketamine in healthy volunteers using positron emission tomography (PET). Eur Neuropsychopharmacol 7: 25-38.

Wilson SM, Isenberg AL, Hickok G (2009). Neural correlates of word production stages delineated by parametric modulation of psycholinguistic variables. Hum Brain Mapp 30: 3596-3608. 